E. M. Fonseca Aizpuru, F. J. Nuño Mateo, L. Otero Guerra', C. López de Mesa ${ }^{2}$

Servicios de Medicina Interna $y^{1}$ Microbiología. ${ }^{2}$ Unidad de Cuidados Intensivos. Hospital de Cabueñes, Gijón. Oviedo

1. Burkert T, Watanakunakorn C. Group A Streptococcal Bacteremia in a Community Teaching Hospital 1980-1989. CID 1992; 14: 29-37.

2. Bosh T, Riera M, Galmés M, Ramírez A, Alberti S. Infecciones invasoras por estreptococos del grupo A: características clínicas y análisis microbiológico de 31 casos. Enferm Infecc Microbiol Clin 2001; 19: 371-375.

3. Barnham M, Weightman N, Anderson A, Pagan F, Chapman S. Review of 17 cases of pneumonia caused by Streptococus pyogenes. Eur J Clin Microbiol Infect Dis 1990; 18: 506-509.

4. Muller MP, Low DE, Green KA, Simor AE, Loeb M, Gregson D et al. Clinical and epidemiologic features of group A streptococcal pneumonia in Ontario, Canada. Arch Intern Med 2003; 24 (163): 467-472.

5. Johnson JL. Pleurisy, fever, and rapidly progressive pleural effusion in a healthy, 29-year-old physician. Chest 2001; 119: 1266-1269.

6. Molina M, Ortega G, Pérez Gracia A, Sáez JA. Empiema por estreptococo betahemolítico del grupo A. Enferm Infecc Microbiol Clin 1990; 8(4): 82.

7. Betriu C, Sánchez A, Gómez M, Crucería A, Picazo J. Antibiotic susceptibility of group a streptococi: a 6-year follow-up study. Antimicrob agents Chemother 1993; 37: 1717-1719.

8. Gamba MA, Martinelli M, Schaad HJ, Streuli RA, DiPersio J, Matter L et al. Familial transmission of a serious disease-producing group A Streptococus clone: Case report and review. CID 1997; 24: 1118-21.

9. Braman SS, Donat WE. Explosive pleuritis: Manifestation of group A beta-hemolytic streptococcal infection. AM J Med 1986; 81: 723-726.

\section{Derrame pericárdico neoplásico infectado por Salmonella enteritidis}

\section{Sr. Director:}

Varón de 77 años que acude a urgencias por anorexia, astenia y disnea progresivamente mayor hasta hacerse de reposo, de unos 7 días de evolución. Entre sus antecedentes personales destacaban una resección transuretral de próstata hacía 4 años por hipertrofia benigna, exfumador muy importante desde hacía 5 años y diagnosticado, hacía 5 meses, de masa en pulmón izquierdo que contactaba con vena pulmonar izquierda, grasa mediastínica y pericardio; se realizó neumonectomía izquierda diagnosticándose de carcinoma epidermoide. Desde entonces precisaba de oxigenoterapia domiciliaria a $2 \mathrm{~L} / \mathrm{min}$. En urgencias en la exploración física destacaba una tensión arterial de 80/40, palidez cutánea, taquipnea, tonos cardíacos rítmicos, muy apagados, junto con taquicardia, así como roce pericárdico y pulso paradójico. La saturación de oxígeno con gafas nasales a $2 \mathrm{~L} / \mathrm{min}$ era del $92 \%$. En la analítica destacaba: 16.680 leucocitos/IL, con un $82 \%$ de neutrófilos, hemoglobina de $10 \mathrm{~g} / \mathrm{dL}$ y LDH de 838 UI/L, con el resto de parámetros del hemograma así como iones, creatinina, CPK, GPT y bilirrubina dentro de límites normales. Con la sospecha de taponamiento cardíaco se realizó ecocardiograma que mostró la presencia de derrame pericárdico severo rodeando todo el corazón, ingresando en planta para tratamiento. En planta se realizó drenaje pericárdico, que se retiró al día siguiente, mandando muestras del líquido extraído a microbiología y anatomía patológica, así como al laboratorio de urgencias, presentando unas proteínas totales de 5,2 g/dL, LDH:10.300 UI/L y 84.410 leucocitos, con $85 \%$ de polimorfonucleares. Tres días después se recibe resultado de microbiología con crecimiento en el líquido pericárdico de abundantes bacilos gram negativos identificados como Salmonella enteritidis, por lo que se inició tratamiento en función del antibiograma con ciprofloxacino intravenoso, a pesar de lo cual el paciente presentó deterioro general progresivo, con reaparición del derrame pericárdico de intensa cuantía, falleciendo 3 días después. El resultado de la citología del líquido pericárdico fue negativo para células malignas. Desde el ingreso el paciente presentó diarrea semilíquida de 2 a 4 deposiciones/día, con coprocultivos, hemocultivos y toxina de Clostridium negativos. Las infecciones cardiovasculares debidas a Salmonella enteritidis son infrecuentes (1), así como las infecciones pericárdicas, suponiendo estas últimas menos del $2 \%$ de las presentaciones extraintestinales (2). Pacientes con derrame pericárdico de base, por ejemplo secundario a uremia están predispuestos a padecerlas, así como pacientes oncológicos o inmunocomprometidos (1,2,5,7). El diagnóstico se basa en cultivos bacteriológicos en sangre y líquido pericárdico (3). El diagnóstico precoz, la rápida instauración del tratamiento antibiótico adecuado así como el drenaje quirúrgico son esenciales para la supervivencia $(4,1)$, siendo documentada en la literatura médica una tasa de mortalidad superior al $50 \%$ a pesar del tratamiento según distintas series $(2,8)$.

\section{E. Alonso Moreno, L. López Cubero, E. M. Aguilar Romo, S. Alexandru, M. Ortega Juaristi, P. García Bermejo, A. Conde Palacios, E. Herrera López}

Servicio de Medicina Interna. Fundación Jiménez Díaz. Clínica de la Concepción. Universidad Autónoma. Madrid

1. Fernández Guerrero ML, Aguado JM, Arribas A, Lumbreras C, de Górgolas M. The spectrum of cardiovascular infections due to Salmonella enterica: a review of clinical features and factors determining outcome. Baltimore: Medicine 2004; 83 (2): 123-38.

2. Ramy Badawi, Thuraia Nageh, David Walker, Richard Wray. Nontyphoidal salmonella pericarditis: a case of Salmonella typhimuriumphage type 2 pericarditis. Intern J Cardiol 2002; 82 (2002): 187-89.

3. Victor F, Gras D, Le Breton H, Gras S, Amelot J, Pony JC. Salmonella enteritidis pericarditis. Apropos of a case and review of the literature. Arch Mal Coeur Vaiss 1997; 90 (2): 301-3.

4. Kiuchi K, Endo T, Nejima J, Okamatsu K, Takayama M, Takano T, Hayakawa H. Purulent pericarditis with tamponade caused by Salmonella enteritidis. Jpn Circ J 1998; 62 (2): 139-41.

5. Arruvito L, Ber MG, Martínez Martínez JA. Purulent pericarditis with pericardial tamponade caused by Streptococcus agalactiae and Salmonella enterica no typhi. B. Aires: Medicina 2004; 64 (4): 340-2.

6. Yoshioka H, Shigemitsu K, Takeuchi M, Mori S, Imaizumi M, et al. Salmonella pericarditis in a patient with primary idiopathic chylopericardium. Jpn J Thorac Cardiovasc Surg 2003; 51 (1): 16-7.

7. García Clemente M, Hidalgo García F, González Budiño T. Derrame pleural neoplásico infectado por Salmonella enteritidis. An Med Interna (Madrid) 2004; 12: 616-7.

8. Cohen L, Bartlett JA, Corey GR. Extra-intestinal manifestations of salmonella infections. Medicine 1987; 66: 349-88.

Neumonitis intersticial como única manifestación clínica en paciente con esclerosis sistémica sin esclerodermia

\section{Sr. Director:}

La esclerosis sistémica (ES) sin esclerodermia (E) es una entidad infrecuente que se caracteriza por esclerosis sistémica progresiva sin afectación cutánea. Presentamos el caso de un paciente con neumonitis intersticial sin afectación cutánea ni otra clínica asociada, con anti RNA polimerasa (RNP) y anti topoisomerasa I ( $\mathrm{Scl}$ 70) positivos. 